\title{
FOTODEGRADAÇÃO DE DUAS ESPÉCIES DE MADEIRAS TROPICAIS: JATOBÁ (Hymenaea courbaril) E TAUARI (Couratari oblongifolia) SUBMETIDAS À RADIAÇÃO ULTRAVIOLETA
}

\author{
Julia de Almeida Costa ${ }^{1}$, Joaquim Carlos Gonçalez ${ }^{2}$, José Arlete Alves Camargos ${ }^{3}$, Igor Alberto Silva Gomes ${ }^{4}$
}

(recebido: 28 de setembro de 2009; aceito: 22 de dezembro de 2010)

\begin{abstract}
RESUMO: Objetivou-se, com este trabalho, acompanhar o processo de fotodegradação da cor natural superficial de madeiras de duas espécies tropicais quando submetidas à radiação artificial ultravioleta. As duas espécies de madeiras pesquisadas foram o jatobá (Hymenaea courbaril) de coloração marrom avermelhada, e tauari (Couratari oblongifolia) de coloração oliva amarelado. As amostras destas madeiras foram submetidas a quatro ciclos de radiação ultravioleta (UV), no comprimento de onda de $350 \mathrm{~nm}$, perfazendo-se um total de 168 horas de irradiação. A mudança da cor natural das madeiras, indicativo do processo de fotodegradação, foi monitorada por espectrocolorimetria. As madeiras das duas espécies sofreram alteração de cor causada pela fotodegradação, havendo um escurecimento dessas, que foi quantificado pela intensidade da luz refletida. $\mathrm{O}$ tauari apresentou-se como a espécie mais resistente à radiação UV e o jatobá apresentou maior tendência para avermelhar-se. Ambas as espécies foram classificadas como madeiras com variação de cor muito apreciável.
\end{abstract}

Palavras-chave: Sistema CIELAB, degradação fotoquímica, madeiras tropicais.

\section{PHOTODEGRADATION OF TWO TROPICAL WOOD SPECIES: JATOBÁ (Hymenaea courbaril) AND TAUARI (Couratari oblongifolia) SUBMITTED TO ULTRAVIOLET RADIATION}

\begin{abstract}
The objective of this study was to follow the process of photodegradation of the natural color of wood surface of two tropical species when exposed to ultraviolet radiation. It were used the wood of jatobá (Hymenaea courbaril) with reddish brown coloration, and tauari (Couratari oblongifolia) with yellowish olive coloration. Samples of these woods were subjected to four cycles of ultraviolet radiation (UV) amounting to a total of 168 hours of irradiation and at $350 \mathrm{~nm}$ of wave length. Changes of the woods natural color, indicative of the photodegradation process, was monitored by spectrocolorimetry. Both species underwent changes in color caused by photodegradation, since the irradiation caused darkening which was quantified by the intensity of reflected light. Tauari demonstrated greater resistance to UV radiation and jatobá showed greater tendency to redden up. Both species were classified as woods with very appreciable variation in color.
\end{abstract}

Key words: CIELAB System, photochemical degradation, tropical woods.

\section{INTRODUÇÃO}

A durabilidade natural da madeira está relacionada à exposição a ambientes internos ou externos, às condições de umidade a que estará submetida, ao uso que se pretende dar à peça e ao acabamento protetor que será aplicado. A exposição da madeira nesses ambientes, particularmente nos externos, torna-a suscetível a uma série de condições que possibilitam sua degradação natural, independente da presença de xilófagos.

Os principais fatores que contribuem para a degradação natural da madeira são: umidade, luz, forças mecânicas e calor; que podem atuar isoladamente ou de forma combinada. A peça de madeira exposta à luz absorve intensamente a radiação solar e sofre degradação fotoquímica devido à ação dos raios ultravioleta, que comprometem o seu aspecto geral, tornando-a descorada ou acinzentada, amarelada ou escurecida, dependendo da influência da composição da madeira, principalmente dos extrativos (AYADI et al., 2003; CHANG et al., 1982; FEIST, 1983). As madeiras analisadas neste trabalho foram a do jatobá (Hymenaea courbaril) e a do tauari (Couratari oblongifolia) que possuem densidade de 0,88 e $0,61 \mathrm{~g} / \mathrm{cm}^{3}$ e teor de extrativos de 8,7 e 1,4\% respectivamente (CENTRO..., 2010; KLITZKE et al., 2008; PASTORE et al., 2008).

A fotodegradação da madeira é um fenômeno superficial, devido à grande quantidade de grupos ou sistemas cromóforos dos componentes distribuídos na

\footnotetext{
'Engenheira Florestal, Mestranda em Ciências Florestais - Universidade de Brasília - Campus Universitário Darcy Ribeiro Cx. P. 04357 - 70919-970 - Brasília, DF - illithien@hotmail.com

2Engenheiro Florestal, Professor Dr. em Ciências Florestais - Universidade de Brasília - Campus Universitário Darcy Ribeiro Cx. P. 04357 - 70919-970 - Brasília, DF-goncalez@unb.br

${ }^{3}$ Administrador, Pesquisador Mestre em Ciências Florestais e Ambientais - Laboratório de Produtos Florestais - Serviço Florestal Brasileiro - 70818-900 - Brasília, DF - jose.camargos@ibama.gov.br

${ }^{4}$ Tecnólogo Agroindustrial, Mestrando em Engenharia Mecânica - Universidade de Brasília - Campus Universitário Darcy Ribeiro Cx. P. 04357 - 70919-970 - Brasília, DF - igor_brunini@hotmail.com
} 
camada externa que impedem a entrada da luz na madeira. De acordo com Hon (2001), citado por Pastore (2004) ao analisar a fotodegradação causada pela radiação ultravioleta em madeiras de cinco espécies tropicais, de forma geral, nas espécies produtoras de madeira, a radiação ultravioleta consegue penetrar até uma profundidade de $75 \mu \mathrm{m}$ e a visível atinge cerca de $200 \mu \mathrm{m}$, dependendo da cor original da madeira. Apesar da radiação visível penetrar mais profundamente, sua energia (inferior a $70 \mathrm{kcal} / \mathrm{mol}$ ) é insuficiente para romper as ligações químicas em qualquer um dos constituintes da madeira. Assim sendo, por possuir uma energia maior (em torno de $90 \mathrm{kcal} / \mathrm{mol}$ ), a radiação ultravioleta é considerada a mais danosa ao uso da madeira.

A lignina é o principal constituinte responsável pela fotodegradação da madeira. Segundo Mitsui e Tsuchikawa (2005), a mudança de cor da madeira pela ação da luz está relacionada à produção de quinonas (extrativos que mudam a cor da madeira) e tem início após a degradação da lignina por oxidação. Como as coníferas normalmente possuem teores mais elevados deste componente, as madeiras de folhosas são consideradas mais resistentes à mudança de cor do que as madeiras de coníferas.

Uma das formas cada vez mais frequentes que vem sendo utilizada para classificar e caracterizar a madeira e os derivados é a colorimetria, que tem como base o sistema CIELAB 1976.

Os espectrofotômetros, segundo Michot (1994), medem a refletância de uma amostra e de um branco padrão nos mesmos comprimentos de onda, o que leva à porcentagem de luz refletida pelo objeto em função de um padrão.

Gonçalez et al. (2001) citam que o sistema CIELAB 1976 fornece um espaço mais uniforme da distribuição das cores. A sensação da cor está baseada em três elementos: a claridade ou luminosidade, a tonalidade ou matiz e a saturação ou cromaticidade.

De acordo com os mesmos autores, a claridade ou luminosidade $\left(\mathrm{L}^{*}\right)$ define a escala padrão cinza entre o branco e o preto variando de 0 (preto total) a 100 (branco absoluto). A tonalidade é dada pelas cores verdevermelho e azul-amarelo, definidas pelas variáveis $a^{*} \mathrm{e}$ $b^{*}$ respectivamente, em que seus valores encontram-se na faixa de 0 a 60 (unidimensional). A tonalidade pode ser dada também pelo ângulo do círculo, interpretada pela variável h*. Ligam-se pontos de cores no espaço CIELAB 1976, com a origem do sistema de coordenadas. O ângulo constituído por esta reta e pelo eixo a* é chamado de $\mathrm{h}^{*}$. A variável $\mathrm{C}$ mostra o valor da saturação ou cromacidade da cor.
Os casos em que C é nulo indicam uma cor que se encontra sobre o eixo $\mathrm{Y}$ e é descrito com a ajuda do valor de L*, chamando-o de cor cinza. Um valor de $\mathrm{C}$ muito elevado indica uma cor fortemente saturada. Quanto mais distante do eixo, mais saturada será a cor. Esta variável também apresenta valores no intervalo de 0 a 60 (unidimensional).

Objetivou-se,com esta pesquisa, verificar o processo de fotodegradação e alteração da cor das madeiras de jatobá (Hymenaea courbaril) e tauari (Couratari oblongifolia), submetidas à radiação ultravioleta artificial.

\section{MATERIAL E MÉTODOS}

Esta pesquisa desenvolveu-se na Universidade de Brasília e no Laboratório de Produtos Florestais (LPF)/ SFB - Brasília DF.

As madeiras de jatobá (Hymenaea courbaril) de coloração marrom avermelhada, e tauari (Couratari oblongifolia) de coloração oliva amarelado são provenientes do mercado madeireiro de Belém (PA). Amostras dessas espécies foram coletadas, ao acaso, em indústrias de pisos e painéis de madeira. Essas amostras foram identificadas para confirmação das espécies.

Para a realização do trabalho, foram confeccionados 4 corpos de prova de cada espécie com dimensões de $80 \times 30 \times 5 \mathrm{~mm}$ (comprimento $\mathrm{x}$ largura $\mathrm{x}$ espessura). Posteriormente, as amostras tiveram sua superfície polida manualmente com uma lixa grana 150 . Os corpos de prova foram retirados, respectivamente, nos sentidos radial e tangencial. Esses eram os sentidos de desdobros que as madeiras se apresentavam na amostragem feita para este trabalho. O teor de umidade dos corpos de prova para a realização do trabalho foi de $12 \%$.

Para simular o efeito da radiação ultravioleta dos raios solares sobre a superfície da madeira, as amostras foram levadas a um reator fotoquímico (Rayonet, USA), com 12 lâmpadas UV de $35 \mathrm{~W}$ cada uma, as quais emitem radiação no comprimento de onda de 350 nanômetros.

As amostras foram fixadas em um carrossel e tiveram o verso da superfície protegido da irradiação. A distância entre os corpos de prova e as lâmpadas foi de aproximadamente $2,0 \mathrm{~cm}$, e a temperatura média dentro do reator, em torno de $37^{\circ} \mathrm{C}$, foi assegurada por um ventilador interno e por refrigeração do ambiente.

O procedimento de simulação da fotodegradação da madeira foi feito por meio de 4 ciclos de $42 \mathrm{~h}$, totalizando $168 \mathrm{~h}$ de irradiação UV. Essa parte da pesquisa foi realizada no Laboratório de Materiais e Combustíveis do Instituto de Química da Universidade de Brasília.

Cerne, Lavras, v. 17, n. 1, p. 133-139, jan./mar. 2011 
Os testes colorimétricos foram realizados no Laboratório de Produtos Florestais do Serviço Florestal Brasileiro e os dados foram obtidos utilizando o espectrofotômetro Datacolor Microflash 200d conectado a um computador. O iluminante utilizado foi o D65 e o ângulo de observação foi de $10^{\circ}$ com luz especular incluída.

Para cada corpo de prova, foram realizadas 15 medições, fazendo-se uma varredura na face radial do tauari e na face tangencial do jatobá. Assim, obtiveram-se os parâmetros colorimétricos: L*, a*, b*, C e h*. Esses parâmetros foram coletados antes e depois dos corpos de prova serem submetidos a cada um dos quatro ciclos da fotodegradação.

Os dados foram processados no software Excel para que as variáveis colorimétricas utilizadas pelo sistema CIELAB 1976 pudessem ser analisadas estatisticamente, aplicando-se ANOVA e teste de média (Tukey), para posteriores comparações descritivas.

As diferenças entre os parâmetros colorimétricos (BILLMEYER; SATZMAN, 1981) foram utilizadas para quantificar a mudança total de cor $\Delta \mathrm{E}^{*}$ por meio da Equação 1:

$\Delta \mathrm{E}^{*}=\left(\Delta \mathrm{L}^{* 2}+\Delta \mathrm{a}^{* 2}+\Delta \mathrm{b}^{* 2}\right)^{1 / 2}$

em que: $\Delta \mathrm{L}^{*}=$ variação da Luminosidade; $\Delta \mathrm{a}^{*} \mathrm{e} \Delta \mathrm{b}^{*}=$ variação das coordenadas cromáticas. Os sinais positivo e negativo fornecidos pelo sistema significam: $+\Delta \mathrm{L}^{*}$ aumento da luminosidade; $-\Delta \mathrm{L} *$ redução da luminosidade; $+\Delta \mathrm{a}^{*}$ aumento na cor vermelha; $-\Delta \mathrm{a}^{*}$ aumento na cor verde; $+\Delta b^{*}$ aumento na cor amarela e $-\Delta b^{*}$ aumento na cor azul.

Cui et al. (2004) utilizaram os valores de variação de cor $\left(\Delta \mathrm{E}^{*}\right)$ da Tabela 1 para classificar a diferença da cor. Esta tabela foi elaborada por Hikita et al. (2001) que se baseou em níveis de percepção.

Tabela 1 - Classificação da variação total da cor $\left(\Delta \mathrm{E}^{*}\right)$ de madeiras.

Table 1 - Total color variation ( $\left.\Delta E^{*}\right)$ classification of woods.

\begin{tabular}{cc}
\hline Variação da cor $\left(\Delta \mathrm{E}^{*}\right)$ & Classificação \\
\hline $0,0-0,5$ & Desprezível \\
$0,5-1,5$ & Ligeiramente perceptível \\
$1,5-3,0$ & Notável \\
$3,0-6,0$ & Apreciável \\
$6,0-12,0$ & Muito apreciável \\
\hline
\end{tabular}

\section{RESULTADOS E DISCUSSÃO}

Os valores médios dos parâmetros colorimétricos das espécies durante os ciclos de exposição UV são apresentados na Tabela 2 .

Tabela 2 - Valores médios dos parâmetros colorimétricos das espécies estudadas.

Table 2 - Average values of the colorimetric parameters of the studied species.

\begin{tabular}{|c|c|c|c|c|c|c|c|c|}
\hline Espécie & Face & $\begin{array}{l}\text { Radiação UV } \\
\text { (horas) }\end{array}$ & $\mathrm{L}^{*}$ & $a^{*}$ & $b^{*}$ & $\mathrm{C}$ & $\mathrm{h}^{*}$ & Nome da cor $(* *)$ \\
\hline \multirow{5}{*}{ Jatobá } & \multirow{5}{*}{ Tangencial } & 0 & $50,12^{1}$ & $13,63^{1}$ & $19,02^{1}$ & $23,41^{1}$ & $54,38^{1}$ & \multirow[t]{4}{*}{ Marrom avermelhado } \\
\hline & & 42 & $44,21^{2}$ & $16,42^{2}$ & $19,92^{2}$ & $25,83^{2}$ & $50,51^{2}$ & \\
\hline & & 84 & $43,16^{3}$ & $16,74^{3}$ & $19,69^{3}$ & $25,85^{3}$ & $49,63^{3}$ & \\
\hline & & 126 & $42,83^{4}$ & $16,82^{4}$ & $19,30^{4}$ & $25,61^{4}$ & $48,93^{4}$ & \\
\hline & & 168 & $43,32^{5}$ & $17,33^{5}$ & $19,51^{5}$ & $26,11^{5}$ & $48,40^{5}$ & Vermelho escuro \\
\hline \multirow{5}{*}{ Tauari } & \multirow{5}{*}{ Radial } & 0 & $64,76^{1}$ & $6,21^{1}$ & $25,54^{1}$ & $26,28^{1}$ & $76,32^{1}$ & \multirow[t]{4}{*}{ Oliva amarelado } \\
\hline & & 42 & $62,81^{2}$ & $7,53^{2}$ & $27,01^{2}$ & $28,04^{2}$ & $74,42^{2}$ & \\
\hline & & 84 & $61,59^{3}$ & $8,08^{3}$ & $28,12^{3}$ & $29,26^{3}$ & $73,96^{3}$ & \\
\hline & & 126 & $60,78^{4}$ & $8,72^{4}$ & $28,79^{4}$ & $30,08^{4}$ & $73,14^{4}$ & \\
\hline & & 168 & $60,65^{5}$ & $9,06^{5}$ & $29,57^{5}$ & $30,93^{5}$ & $72,97^{5}$ & Marrom oliva \\
\hline
\end{tabular}

Dentro da mesma espécie, os valores assinalados com números diferentes na mesma coluna diferem entre si ao nível de $1 \%$ de probabilidade pelo teste de Tukey;

$(* *)=$ Cor natural da madeira, segundo classificação proposta por Camargos e Gonçalez (2001). 
De uma forma geral, para todos os tratamentos e para as duas espécies, as coordenadas $\mathrm{a}^{*} \mathrm{e} \mathrm{b}^{*}$ são responsáveis pela formação da cor das madeiras. No entanto, segundo a literatura (CAMARGOS; GONÇALEZ, 2001; GONÇALEZ et al., 2001; GONÇALEZ; MACEDO, 2003), a coordenada $\mathrm{a}^{*}(+)$ (coloração vermelha) acima de 10 manifesta-se de forma mais pronunciada em relação a coordenada $b^{*}$, predominando a nuance avermelhada na formação da cor dessas madeiras. Assim, para a espécie jatobá, a coordenada a* (coloração vermelha) tem uma presença mais marcante na composição da cor, sendo a principal variável na formação final da cor desta madeira. Já para a espécie tauari, a principal variável colorimétrica é coordenada b* (coloração amarela) que apresenta valores mais elevados quando comparados à coordenada $\mathrm{a}^{*}$. Para as duas espécies, o ângulo de tinta $\mathrm{h}^{*}$ (sistema CIELAB) confirma essa constatação, colocando a madeira de jatobá mais próximo do eixo vermelho e a madeira de tauari próximo ao eixo amarelo.

Comparando os tratamentos (Tabela 2), verifica-se que,para as duas espécies, todos os parâmetros colorimétricos mostraram diferenças estatísticas significativas. Em ambas as espécies, há uma tendência de escurecimento da madeira com o aumento do tempo de radiação ultravioleta. A coordenada cromática $\mathrm{a}^{*}$ mostra tendência de aumentar, sendo a principal responsável pelo escurecimento das madeiras. Novamente, o ângulo de tinta $\left(\mathrm{h}^{*}\right)$ corrobora para o entendimento da coloração mais escura das madeiras. A diminuição da luminosidade na madeira de jatobá e o aumento da pigmentação vermelha (coordenada $a^{*}$ ) altera a sua cor para vermelho escuro. Observa-se ainda que a madeira de tauari tem uma cor mais saturada (variável C) provavelmente dada pela pigmentação amarela $\left(b^{*}\right)$ mais pronunciada nesta espécie. A diminuição da claridade desta espécie deixa-a com uma coloração marrom oliva.

As variações dos parâmetros colorimétricos provocadas pelos quatro ciclos de radiação UV, totalizando 168 horas de radiação, encontram-se na Tabela 3.

É possível verificar, a partir dos valores negativos de $\Delta \mathrm{L}^{*}$, que houve um escurecimento na superfície das duas espécies ao final dos quatro ciclos de radiação UV. Observa-se que, para a madeira do jatobá, após cerca de 126 horas de radiação, houve uma inversão da declividade do $\Delta \mathrm{L}^{*}$, sugerindo o final do processo de escurecimento da madeira e possivelmente uma estabilização (Figura 1). Comportamento semelhante foi observado por Silva e Pastore (2004), que verificaram ainda que esse processo de clareamento com madeiras avermelhadas tende a estabilizar-se à medida que aumenta o tempo de tratamento.
Tabela 3 - Variações dos parâmetros de cor na superfície das madeiras estudadas.

Table 3 - Change in color parameters in surfaces of the studied woods.

\begin{tabular}{cccccc}
\hline \multirow{2}{*}{ Parâmetro } & \multirow{4}{c}{ Espécie } & \multicolumn{3}{c}{ Tempo de radiação UV (horas) } \\
\cline { 3 - 6 } & & $42 \mathrm{~h}$ & $84 \mathrm{~h}$ & $126 \mathrm{~h}$ & $168 \mathrm{~h}$ \\
\hline \multirow{2}{*}{$\Delta \mathrm{L}^{*}$} & Jatobá & $-5,91$ & $-6,96$ & $-7,29$ & $-6,80$ \\
& Tauari & $-1,95$ & $-3,17$ & $-3,98$ & $-4,11$ \\
\multirow{2}{*}{$\Delta \mathrm{a}^{*}$} & Jatobá & 2,79 & 3,11 & 3,19 & 3,70 \\
& Tauari & 1,32 & 1,87 & 2,51 & 2,85 \\
\multirow{2}{*}{$\Delta \mathrm{b}^{*}$} & Jatobá & 0,90 & 0,67 & 0,28 & 0,49 \\
& Tauari & 1,48 & 2,59 & 3,25 & 4,04 \\
\multirow{2}{*}{$\Delta \mathrm{E}^{*}$} & Jatobá & 6,60 & 7,65 & 7,96 & 7,75 \\
& Tauari & 2,78 & 4,50 & 5,72 & 6,43 \\
\hline
\end{tabular}

$\Delta \mathrm{L}^{*}=$ variação da luminosidade; $\Delta \mathrm{a}^{*}=$ variação na coordenada cromática verde-vermelha; $\Delta \mathrm{b}^{*}=$ variação na coordenada cromática amarela-azul; $\Delta \mathrm{E}^{*}$ = variação total da coloração.

Estudos feitos com madeiras de clima temperado (MÜLLER et al., 2003) e tropical (NZOKOU; KAMDEM, 2002) também relatam esse comportamento. $\mathrm{O}$ escurecimento inicial é atribuído à formação de compostos quinoidais em função, sobretudo, da degradação da lignina pela radiação UV na presença de oxigênio (HON, 2001).

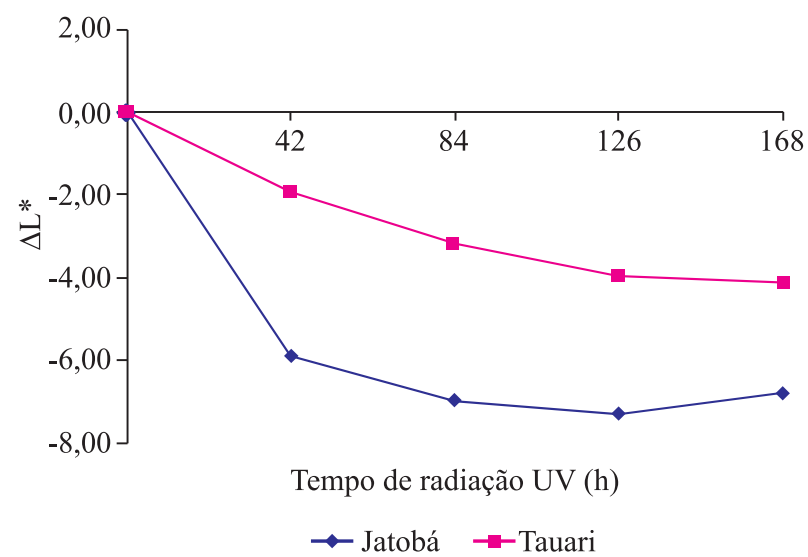

Figura 1 - Curvas de variação de luminosidade $\left(\Delta \mathrm{L}^{*}\right)$ do jatobá e do tauari provocada pela fotodegradação.

Figure 1 - Curves of variation of luminosity $\left(\Delta L^{*}\right)$ of jatobá and tanari woods caused by photodegradation.

Nas condições desse ensaio, o tauari sofreu menor mudança na cor vermelha $\left(\Delta \mathrm{a}^{*}=2,85\right)$, apresentando

Cerne, Lavras, v. 17, n. 1, p. 133-139, jan./mar. 2011 
maior estabilidade em relação a essa tonalidade, e o jatobá apresentou maior estabilidade para o amarelo já que esta coordenada cromática foi a que variou menos $\left(\Delta \mathrm{b}^{*}=\right.$ $0,49)$. Os resultados encontrados por Pastore (2004) para o jatobá demonstram a maior tendência desta espécie para avermelhar-se com o decorrer do tempo.

A variação total da cor $\left(\Delta \mathrm{E}^{*}\right)$, determinada pela equação 1, leva em consideração as diferenças das coordenadas cromáticas e de luminosidade, fornecendo assim, uma visão mais ampla sobre o desempenho das madeiras sob a ação de fotodegradação. A figura 2 ilustra o comportamento das duas espécies estudadas.

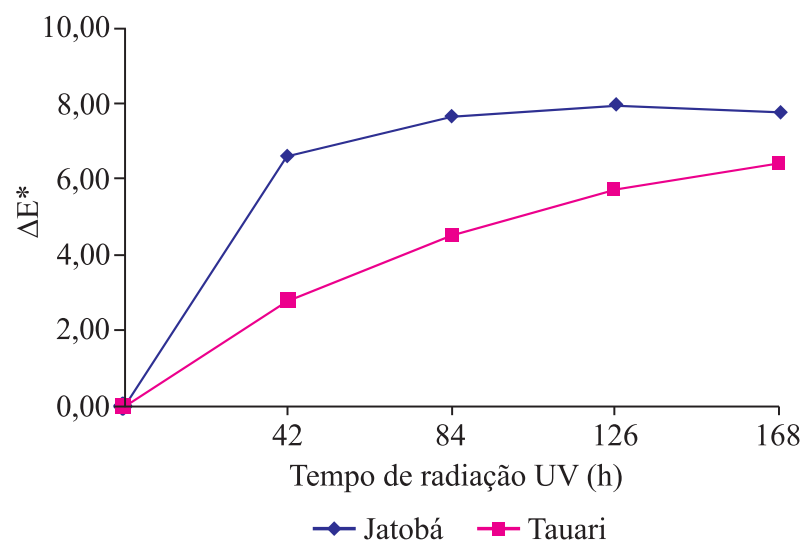

Figura 2 - Curvas de variação total de cor $\Delta \mathrm{E}^{*}$ causada pela fotodegradação do jatobá e do tauari.

Figure 2 - Curves of total color variation $\Delta E^{*}$ caused by photodegradation of jatobá and tauari.

O desempenho do $\Delta \mathrm{E}^{*}$ para as duas espécies apresentou um comportamento semelhante, principalmente durante as primeiras horas de radiação, em que ocorreram as maiores alterações de cor. A variação da cor, no final do último tratamento, $\Delta \mathrm{E}^{*}=7,75$ e $\Delta \mathrm{E}^{*}=6,43$, para o jatobá e tauari, respectivamente, foi classificada como muito apreciável, segundo a classificação proposta por Hikita et al. (2001). Barreto e Pastore (2009), Pastore (2004) e Silva et al. (2007), classificaram o $\Delta \mathrm{E}^{*}$ dessas espécies como apreciável e notaram que as curvas tendem a estabilizar-se com o tempo de radiação, formando um patamar. A diferença de classificação do $\Delta \mathrm{E}^{*}$,encontrada neste trabalho, em relação à classificação feita pelos autores mencionados, pode ser atribuída principalmente à diferença na quantidade e nos tipos de extrativos presentes nas madeiras estudadas, e também à variação da distribuição desses extrativos através da madeira.
A refletância das madeiras estudadas em função do comprimento de onda pode ser observada na Figura 3. A madeira do jatobá reflete menos luz que o tauari em todos os comprimentos de onda do espectro.

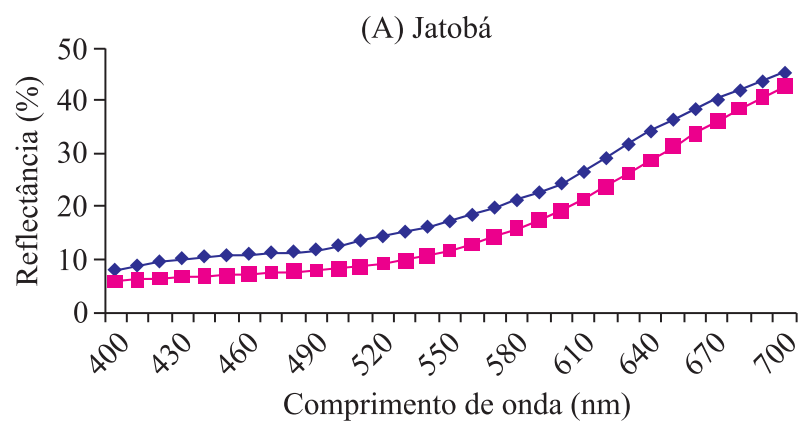

(B) Tauari

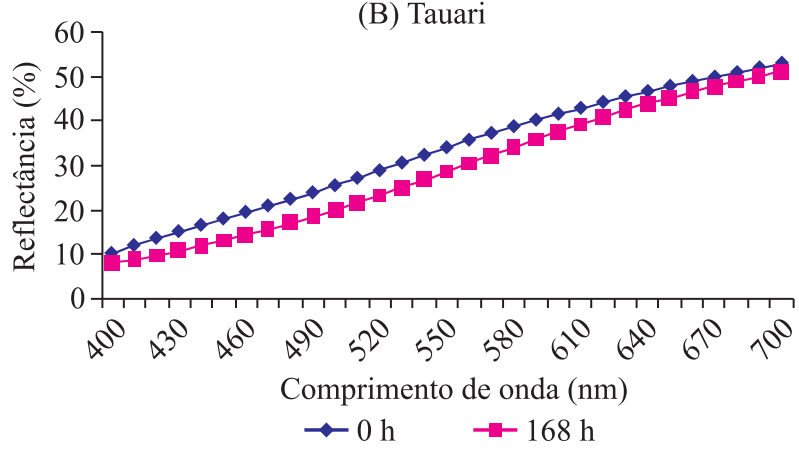

Figura 3 - Refletância das cores em função do comprimento de onda das madeiras do jatobá (A) e do tauari (B), antes e após $168 \mathrm{~h}$ de radiação UV.

Figure 3 - Reflectance of colors according to the wavelength of the jatobá (A) and Tauari (B) wood before and after 168h of UV radiation.

É possível visualizar um comportamento padrão nas curvas de refletância das duas espécies, uma vez que a irradiação UV provocou escurecimento na cor das amostras, levando à diminuição na intensidade da luz refletida. Além de as curvas de refletâncias serem apropriadas para acompanhar as modificações colorimétricas das madeiras causadas pela irradiação UV, estas também podem ser empregadas para acompanhar as mudanças químicas que ocorrem durante o processo.

\section{CONCLUSÕES}

A espectrocolorimetria permitiu obter uma visão geral do comportamento das madeiras tropicais investigadas, quando submetidas à irradiação UV artificial.

Cerne, Lavras, v. 17, n. 1, p. 133-139, jan./mar. 2011 
As madeiras das duas espécies estudadas sofreram alteração de cor causada pela fotodegradação, e ambas foram classificadas como madeiras com variação de cor muito apreciável. O jatobá apresentou maior tendência para avermelhar-se, enquanto o tauari apresentou coloração oliva escura.

Os resultados obtidos para o tauari sugerem que esta madeira apresenta maior resistência à radiação UV do que o jatobá, que também é indicada para uso externo.

As diferenças de comportamento das cores das madeiras investigadas possuem como fatores entre outros, a variação do conteúdo e composição de extrativo de cada espécie.

Essas informações mostram que as madeiras destas espécies, irão sofrer alterações em suas cores com o passar do tempo. Certamente, o custo suplementar dos industriais para mitigar esse processo será compensado por uma maior agregação de valores ao produto comercializado, uma vez que o material será mais homogêneo, mostrando qualidade superior.

\section{REFERÊNCIAS}

AYADI, N.; LEJEUNE, F.; CHARRIER, F.; CHARRIER, B.; MERLIN, A. Color stability of heat-treated wood during artificial weathering. Holz als Roh und Werkstoff, v. 61, n. 3, p. 221-226, June 2003.

BARRETO, C. C. K.; PASTORE, T. C. M. Resistência ao intemperismo artificial de quatro madeiras tropicais: o efeito dos extrativos. Ciência Florestal, Santa Maria, v. 19, n. 1, p. 23-30, mar. 2009.

BILLMEYER, J. F. W.; SATZMAN, M. Principles of colour technology. New York: J. Wiley, 1981. 240 p.

CAMARgOS, J. A. A.; GONÇALEZ, J. C. A colorimetria aplicada como instrumento na elaboração de uma tabela de cores de madeira. Brasília: MMA, 2001. (Brasil Florestal, 71).

CENTRO das Indústrias Produtoras e Exportadoras de Madeira do Estado de Mato Grosso. Disponível em: $<\underline{\text { http:// }}$ www.cipem.org.br/catalogo/paginas/tauari.html $>$. Acesso em: 10 maio 2010.

CHANG, S. T.; HON, D. N. S.; FEIST, W. C. Photodegradation and photoprotection of wood surfaces. Wood and Fiber, v. 14, n. 2, p. 104-107, 1982.

Cerne, Lavras, v. 17, n. 1, p. 133-139, jan./mar. 2011
CUI, W.; KAMDEM, P.; RYPSTRA, T. Diffuse reflectance infrared Fourier transform spectroscopy (DRIFT) and color changes of artificial weathered wood. Wood and Fiber Science, Madison, v. 36, n. 3, p. 291-301, July/Sept. 2004.

FEIST, W. C. Weathering and protection of wood. In: ANNUAL MEETING OF THE AMERICAN WOODPRESERVERS' ASSOCIATION, 79., 1983, Kansas City. Proceedings... Kansas City, 1983. p. 195-205.

GONÇALEZ, J. C.; JANIN, G.; SANTORO, A. C .S.; COSTA, A. F.; VALLE, A. T. Colorimetria quantitativa: uma técnica objetiva de determinar a cor da madeira. Brasília: MMA, 2001. (Brasil Florestal, 72).

GONÇALEZ, J. C.; MACEDO, D. G. Colorimetria aplicada a madeira de eucalipto. Belo Horizonte: MADETEC, 2003.

HIKITA,Y.; TOYODA, T.; AZUMA, M. Weathering testing of timber: discoloration. In: IMAMURA, Y. High performance utilization of wood for outdooor uses. Kyoto: Press-Net, 2001 .

HON, D. N. S. Weathering and photochemistry of wood. In: HON, D. N. S.; SHIRAISHI, N. Wood and cellulosic chemistry. 2. ed. New York: M. Dekker, 2001.

KLITZKE, R. J.; SAVIOLI, D. L.; BOLZÓN DE MUÑIZ, G. I.; BATISTA, D. C. Caracterização dos lenhos de cerne, alburno e transição de jatobá (Hymenaea sp.) visando ao agrupamento para fins de secagem convencional. Scientia Forestalis, Piracicaba, v. 36, n. 80, p. 279-284, dez. 2008.

MICHOT, S. Classification subjective des parquets de chêne: rapport de stage. Epinal: Université de Nancy, 1994. $48 \mathrm{p}$.

MITSUI, K.; TSUCHIKAWA, S. Low atmospheric temperature dependence on photodegradation of wood. Journal of Photochemistry and Photobiology B: Biology, Lausanne, v. 81, p. 84-88, 2005.

MÜLLER, U. et al. Yellowing and IR-changes of spruce wood as result of UV-irradiation. Journal of Photochemistry Photobiology B: Biology, Lausanne, v. 69, n. 2, p. 97-105, 2003. 
NZOKOU, P.; KAMDEM, D. P. Weathering of two hardwoods species: african paudak (Pterocarpus soyauxii) and red maple (Acer rubum). Journal of Tropical Forest Products, Kuala Lumpur, v. 8, n. 2, p. 200-209, 2002.

PASTORE, T. C. M. Estudos do efeito da radiação ultravioleta em madeiras por espectroscopias RAMAN (FT-RAMAN), de refletância difusa no infravermelho (DRIFT) e no visível (CIE-L*a*b*). 2004. 131 p. Tese (Doutorado) - Universidade de Brasília, Brasília, 2004.

PASTORE, T. C. M.; OLIVEIRA, C. C. K.; RUBIM, J. C.; SANTOS, K. S. Efeito do intemperismo artificial em quatro madeiras tropicais monitorado por espectroscopia de infravermelho (DRIFT). Química Nova, São Paulo, v. 31, n. 8, 2008.

SILVA, J. O.; PASTORE, T. C. M. Fotodecomposição e proteção de madeiras tropicais. Floresta e Ambiente, Seropédica, v. 21, n. 2, p. 7-13, 2004.

SILVA, J. O.; PASTORE, T. C. M.; PASTORE JÚNIOR, F. Resistência ao intemperismo artificial de cinco madeiras tropicais e de dois produtos de acabamento. Ciência Florestal, Curitiba, v. 17, p. 17-23, 2007. 